\title{
Perceptual Effects of Damped and Exaggerated Facial Motion in Animated Characters
}

\author{
Jennifer Hyde ${ }^{1}$, Elizabeth J. Carter ${ }^{2}$, Sara Kiesler ${ }^{3}$, and Jessica K. Hodgins ${ }^{1,2}$ \\ \{jdtam, ejcarter, kiesler, jkh\}@cs.cmu.edu
}

\begin{abstract}
Common guidelines followed in the animation community include the idea that cartoon characters should be exaggerated to better convey emotion and intent, whereas more realistic characters should have "matching" realistic motion. We investigated the effects of rendering style and amount of facial motion on perceptions of character likeability, intelligence, and extraversion. We used cartoon and more realistic-looking characters that were animated with tracked actor motion. The motion was exaggerated and damped in $10 \%$ increments up to a $40 \%$ difference from the original motion. We discovered that motion changes $\pm \mathbf{2 0} \%$ from original motion affected perceptions of likeability and intelligence differently in the realistic-looking and cartoon characters. The realistic-looking characters benefited from increased motion whereas the cartoon characters benefitted from damped motion. Furthermore, the amount of facial motion and perceptions of extraversion were significantly correlated.
\end{abstract}

\section{INTRODUCTION}

We encounter animated characters in many different domains, including entertainment, education, and therapy. These characters need to embody certain attributes to be effective. For example, a virtual peer working with a child with Autism Spectrum Disorder should be engaging and friendly [1]. A virtual assistant or therapist should be intelligent and trustworthy. Characters in games, movies, and television shows need to embody many other personality traits to be believable. Currently, many of the design decisions regarding character appearance and motion are made by artists. Artists follow guidelines as well as their own intuition when animating characters [2], [3].

We are interested in scientifically exploring some of these guidelines and intuitions to better inform animation and design decisions. Specifically, we are interested in understanding the relationship between the amount of character facial motion and the character rendering style. We conducted a controlled laboratory experiment in which participants watched animations of cartoon and more realistic-looking characters reading unique stories, each with a different amount of facial motion. We modified the spatial motion to exaggerate and damp facial motion. Participants then rated each character on questions concerning likeability, trustworthiness, intelligence, and extraversion. In general, participants liked the realistic characters more than the cartoon characters. This preference was amplified when the realistic characters were exaggerated and the cartoon characters were

\footnotetext{
${ }^{1}$ Computer Science Department, ${ }^{2}$ Robotics Institute, ${ }^{3}$ Human Computer Interaction Institute at Carnegie Mellon University, 5000 Forbes Avenue, Pittsburgh, PA 15213, U.S.A.
}

damped. Exaggeration improved perceptions of intelligence for the realistic characters, and damped motion improved perceptions of intelligence for the cartoon characters. We also found that motion level and perceptions of extraversion are positively correlated.

\section{RELATED WORK}

Exaggerated movement is a signature of cartoon-style motion, and this characterization has been widely accepted as a guiding principle of animators who try to create the "illusion of life" [2], [3], [4]. Exaggeration is used to make movement, intention, and emotion more salient. This animation principle has even been borrowed by the robotics community to make human-robot interaction more intuitive and engaging [5], [6], [7]. As characters have become more visually realistic, there has been more demand that their motion also be more realistic [4]. Motion capture is often employed to transfer realistic motion to realistic characters, but the results are sometimes unappealing and uncanny, particularly for facial animation.

Not only are animated characters used for entertainment purposes, but they can also be used at work. In a corporate setting, Inkpen and Sedlins [8] found that users were more particular about their avatar's appearance than the appearance of the avatar with which they interacted. The researchers found that $65 \%$ of participants believed it was important for avatars to convey their user's personality.

McDonnell and colleagues [9] investigated the effect of rendering style and motion on perceptions of a virtual human's appeal, friendliness, and trustworthiness. Participants saw still images of a character in different rendering styles or they saw short clips (6-10 seconds) of the animated character in different rendering styles. Motion did not have a significant effect on appeal, friendliness, or trustworthiness; however, rendering style had a significant effect on all three traits. The characters rendered in the most appealing styles were also rated as the friendliest and most trustworthy. Realistic and cartoon characters were equally well-liked, but characters that were in the middle of the cartoon-to-real spectrum were not well-liked.

We further this research [9] by changing the amount of motion in the animations. Additionally, we present longer clips so that our participants have more time to form their impressions of the characters. 


\section{HYPOTHESES}

It is believed that character rendering style and motion level should "match"; therefore our first hypothesis (H1) is that participant ratings of character likeability and intelligence will be higher for characters with matching rendering style and motion level than for characters with mismatched rendering style and motion level: (H1a) With exaggerated motion, cartoon characters will be rated higher than realisticlooking characters; (H1b) With damped motion, realisticlooking characters will be rated higher than cartoon characters; (H1c) For realistic-looking characters, damped motion will be rated higher than exaggerated motion; (H1d) For cartoon characters, exaggerated motion will be rated higher than damped motion.

Prior research found that extroverts had faster body movement times than introverts [10], [11], [12] suggesting that we should find a positive correlation between motion level and ratings of extraversion $(\mathrm{H} 2)$.

The relationship between extraversion and intelligence is unclear as there is work supporting a positive correlation [10], [13] and more recent work supporting a negative correlation [14]. We expect our results to support a negative correlation between extraversion and intelligence $(\mathrm{H} 3)$.

Finally, research on expert witnesses in court found that likeability and trustworthiness were positively correlated [15]; therefore we expect to find that well-liked animated characters will also be rated as trustworthy (H4).

\section{Procedure And Methods}

We examined participants' impressions of animated characters in a controlled laboratory experiment. Participants viewed animated realistic and cartoon characters with differing amounts of motion. Participants viewed each character once as he/she told a unique story. Each participant saw each motion level only once. We discovered that perceptions of likeability and intelligence differ depending on character rendering style and motion level. We also confirmed that perceptions of likability and trustworthiness are correlated and that amount of motion is positively correlated with perceptions of extraversion.

\section{A. Participants}

We advertised our study on a university experiment scheduling site. Thirty-four adults participated in this study (age range: 18-62 years; median age: 22.5 years; 18 females). We eliminated data from two participants due to equipment problems. All participants read and signed informed consent forms approved by the Institutional Review Board. Participants were compensated for their time.

\section{B. Surveys and Questionnaires}

Participants completed the Ten Item Personality Inventory (TIPI) prior to seeing any animations [16]. After each animation, participants completed a questionnaire asking for their impressions of the character they had just seen. We asked twelve questions on these topics in the form of fivepoint rating scales. Principal component analysis followed by a factor rotation indicated that the twelve questions loaded onto three factors with acceptable reliability (Cronbach's $\alpha$ ).

- Likeability: Five questions on perceived likeability, trustworthiness, sincerity, reliability, and warmth loaded onto this measure. (Cronbach's $\alpha=0.81$ )

- Intelligence: Three questions on perceived intelligence, competence, and how well informed the character was loaded onto this measure. (Cronbach's $\alpha=0.76$ )

- Extraversion: Four questions on perceived extraversion, inhibition (reverse scored), sociability, and dramaticism loaded onto this measure. (Cronbach's $\alpha=0.71$ )

We presented all questionnaires and study stimuli on Apple 27-inch flat panel LED cinema displays connected to machines running OSX 10.6, Matlab, and the Psychophysics Toolbox extensions [17], [18], [19]. Participants wore headphones and input their responses with a keyboard.

\section{Characters and Animations}

We created eight different characters, four of which were in a cartoon style (toon) and the other four in a more realistic style (CG). There were an equal number of male and female characters per rendering style. Characters of the same gender differed by skin tone, hair color, eye color, and shirt color (Fig. 1). Animations were $1086 \times 639$ pixels large and presented at 60 frames per second.

We recorded an actor and actress, from the shoulders up, reading eight short stories using a Sony PMW-EX3 camera and an Audio Technica shotgun microphone. The stories ranged in length from 1:24 to 1:42 (min:s). We used the recordings to create models of the actors' faces, and then we retargeted the actors' motion to our characters.

We used 2D Active Appearance Models (AAMs) to track the actors and synthesize the animated faces [20], [21], [22]. An AAM consists of two independent models that describe shape and appearance variation. We used these models to define all possible face shapes and appearances for our actors and characters. Our face shapes were vectors of 79 coordinates $\left(\mathbf{s}=\left(x_{1}, y_{1}, \ldots x_{79}, y_{79}\right)^{T}\right)$. We created the shape model with hand-labeled training videos. The shape model is defined in Eq. (1) where $\mathbf{s}$ is a new shape, $\mathbf{s}_{\mathbf{0}}$ is the mean shape, and the vectors $\mathbf{s}_{\mathbf{1}}$ through $\mathbf{s}_{\mathbf{m}}$ are the largest basis vectors which span the shape space. The shape

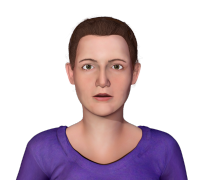

(a) CG Female I

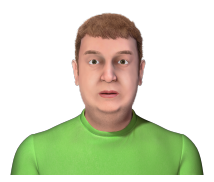

(e) CG Male I

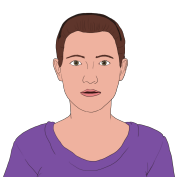

(b) Toon Female I

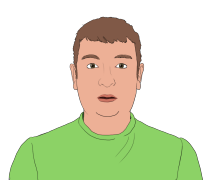

(f) Toon Male I

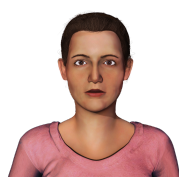

(c) CG Female II

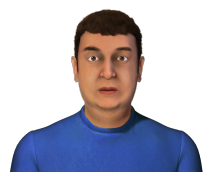

(g) CG Male II

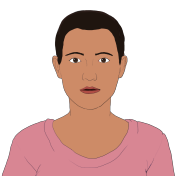

(d) Toon Female II

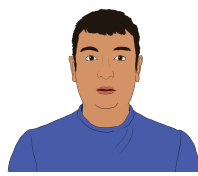

(h) Toon Male II
Fig. 1. The eight characters in cartoon and realistic rendering styles. 
parameters, $p_{i}$, indicate how much each corresponding basis vector contributes to the overall face shape.

$$
\mathbf{S}=\mathbf{s}_{\mathbf{0}}+\sum_{i=1}^{m} \mathbf{s}_{\mathbf{i}} p_{i}
$$

The appearance model is defined similarly in Eq. (2) with appearance, $\mathbf{x}=(x, y)^{T}$, defined as the pixels that lie within the mean face shape. $A(\mathbf{x})$ is the new appearance, $A_{0}(\mathbf{x})$ is the mean appearance, $A_{1}(\mathbf{x})$ through $A_{l}(\mathbf{x})$ are the largest bases spanning the appearance space, and the $\lambda_{i}$ appearance parameters indicate the amount that each appearance base contributes to the new appearance.

$$
A(\mathbf{x})=A_{0}(\mathbf{x})+\sum_{i=1}^{l} \lambda_{i} A_{i}(\mathbf{x}) \quad \forall \mathbf{x} \in \mathbf{s}_{\mathbf{0}}
$$

We followed the procedure from Boker, Theobald, and colleagues [23], [24] to exaggerate and damp the facial motion of the characters. By multiplying the face shape variation by values greater than 1 , the motion is exaggerated, and by multiplying the face shape variation by values less than 1 , the motion is damped. We did not track body motion, so the torsos of our animated characters move rigidly with respect to a pivot located at their mouths. Our characters always face forward as they are created from 2D data. We added rigid points around the tops of the characters' heads to prevent warping, and we damped the face border and nose points by $50 \%$ to ensure that the characters' faces and noses did not appear to be squished or stretched whenever the actors turned their heads slightly.

Each actor's motion was retargeted to characters of the same gender. We did not modify the duration of motion even though our manipulations did change spatial and temporal motion. In other words, the time it took an actor to start and end a motion, such as opening and closing his mouth, was the same regardless of manipulation; however, the actor's smile would be bigger and his lips would move faster in the case of exaggeration. Thus, we used the same audio across all animations that were created from a single actor's recording of a specific story.

\section{Story Calibration}

A single author wrote 24 short stories from the same point of view. We calibrated the stories on interest and emotional complexity, positivity, and intensity through Amazon's Mechanical Turk. We collected 16 to 21 independent ratings for each story. We used a one-way analysis of variance (ANOVA) on emotional complexity followed by pairwise t-tests to find 18 statistically similar stories. Five stories were eliminated for exemplifying negative emotion. We then plotted the thirteen stories that were left and selected the eight stories that were most similar with respect to emotional intensity and interest. To avoid repeating stories, we used an equal number of stories and characters.

\section{E. Motion Levels}

We ran a pretest with twenty additional participants to determine when exaggerated and damped motion with a cartoon character would be noticeably different than the original motion. We tested eight motion levels ranging between $20 \%$ and $180 \%$ of original motion $(20 \%, 40 \%, 60 \%$, $80 \%, 100 \%, 120 \%, 140 \%, 160 \%, 180 \%)$. Participants saw two animations side by side that only differed by motion level. One animation was always $100 \%$ motion, and the other animation was damped or exaggerated. Participants saw the $100 \%$ motion on the left or right side randomly, so that there were no left or right biases in our results. Participants decided whether the characters were moving the same amount or different amounts. Participants pressed 1 , 2 , or 3 on a keyboard where 1 indicated that the left-most animated character was moving more, 2 indicated that the right-most character was moving more, and 3 indicated that the characters were moving the same amount. There was a significant effect of motion level on participant accuracy and response time at the $p<0.0001$ level, $F(7,152)=7.70$ and $F(7,152)=5.37$, respectively. Participants performed above chance at all motion levels except the $120 \%$ level. Accuracy at the $140 \%$ and higher levels was significantly higher than accuracy at the $120 \%$ level. Similarly, accuracy at the $60 \%$ and lower levels was significantly better than at the $80 \%$ level. These results indicate that motion at and below $60 \%$ and at and above $140 \%$ are noticeably damped and exaggerated. Alternatively, motion levels including and between $80 \%$ and $120 \%$ were not noticeably different from the original motion, as depicted in Fig. 2.

We ran another pretest with twenty different participants using a realistic character. This time we tested motion levels in $5 \%$ increments from $30 \%$ to $170 \%$. We ran a one-way ANOVA on participant accuracy and found a significant effect of motion level $(F(27,907)=22.72, p<0.0001)$. Participants performed above chance at all motion levels except those between and including $85 \%$ and $110 \%$. Participants were extremely accurate at motion levels at and below $60 \%$ and at and above $140 \%$, as depicted in Fig. 2. Based on the results from these two pretests, we chose to use the following eight motion levels: $60 \%, 70 \%, 80 \%, 90 \%, 110 \%, 120 \%$, $130 \%$, and $140 \%$. We provide a sample frame at each of these motion levels in Fig. 3.

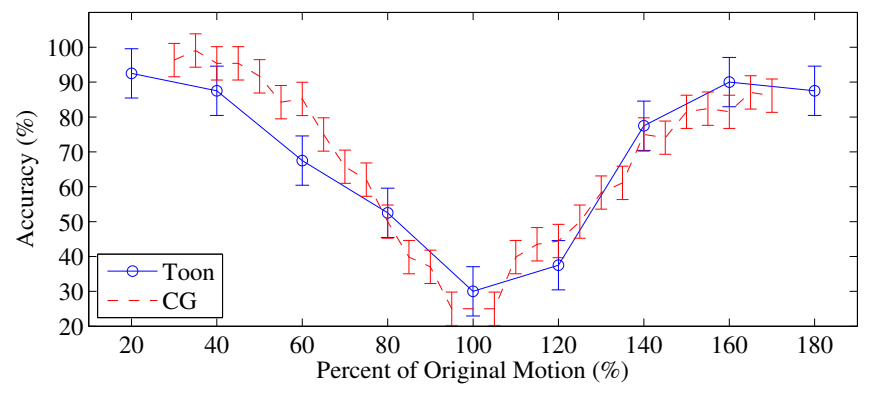

Fig. 2. Motion level pretest results. 


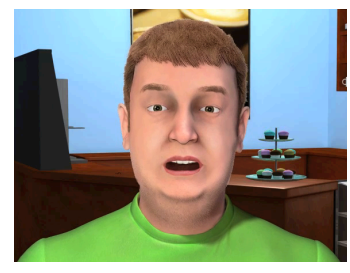

(a) $60 \%$

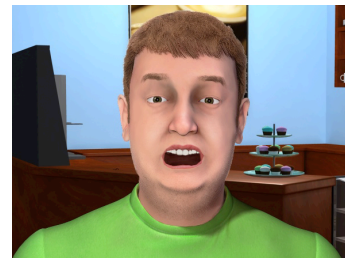

(e) $110 \%$

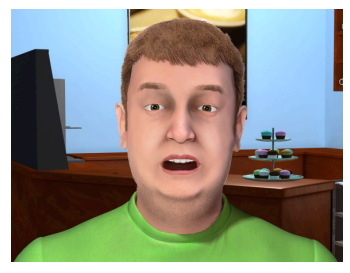

(b) $70 \%$

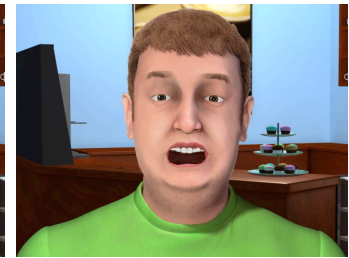

(f) $120 \%$

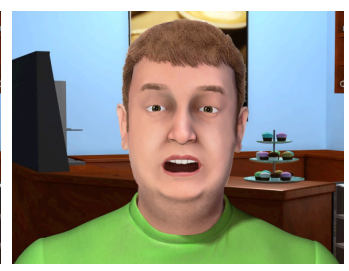

(c) $80 \%$

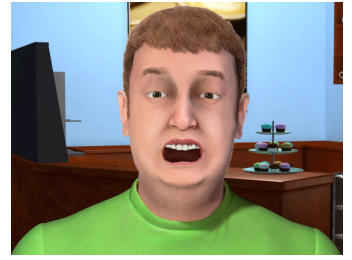

(g) $130 \%$

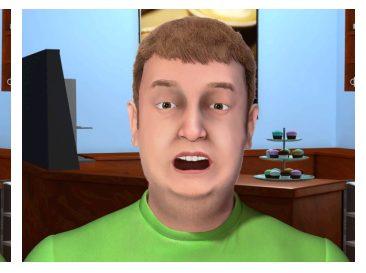

(d) $90 \%$

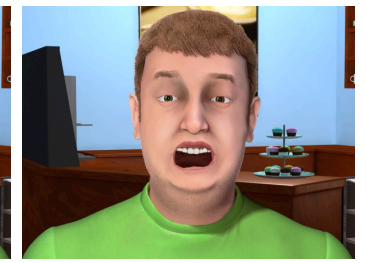

(h) $140 \%$

Fig. 3. Sample still frames of the eight different motion levels.

\section{F. Experimental Design}

We used a repeated-measures experimental design. Motion level and rendering style were within-subjects factors with eight and two conditions, respectively. Because we wanted participants to form impressions of the characters' personalities, we did not allow participants to see any character, motion level, or story more than once. Therefore, participants viewed eight different characters, each displaying a different level of motion, and each telling a different story. We constructed paired orthogonal Latin squares, which simultaneously counterbalanced immediate sequential effects and pairing of conditions [25]. This pair of Latin squares was used to select participants' trial conditions and trial order. Across all participants, every (character, motion) pair occured twice, and the order was counterbalanced to control for possible order effects.

\section{G. Procedure}

Participants arrived at the study location and completed consent forms. An experimenter was present to answer questions and explain the study tasks to participants. The experimenter led participants to the study setup, and participants completed the TIPI. After completion, participants advanced to another instruction screen explaining that they would see a series of animations, each of which would be followed by a questionnaire asking about their impressions of the character in the animation. The participants then viewed each animation and answered the accompanying questions. A screen before each animation reminded participants that they could take a break. At the end of the study the participants were shown a thank you screen, and then the experimenter debriefed and paid them. The experiment took no longer than 40 minutes. The experimenter stayed near the participant during the study so that participants could ask questions or take breaks at any time.

\section{RESUlts}

We conducted a repeated-measures ANOVA to analyze the possible effects of motion level and rendering style on our measures of likeability, intelligence, and extraversion. We found that motion level and rendering style influence participant impressions, although not always in ways that we expected. We also found a significant main effect of rendering style on impressions of character likeability and intelligence. As expected, we found a relationship between motion level and extraversion.

Hypothesis $\mathrm{H} 1$ that character rendering style and motion level should match based on animation principles was only partially supported. The interaction between motion level and rendering style was marginal for likeability and significant for intelligence, $F(7,211)=1.78, p=0.092$ and $F(7,207)=$ $2.91, p=0.006$, respectively (see Fig. 4). We investigated these interactions in more detail with post hoc contrast tests.

While investigating $\mathrm{H} 1 \mathrm{a}$, we found that exaggerated cartoon characters were rated equal to or lower than exaggerated realistic-looking characters. The difference in likeability ratings was not significant when examining all levels of exaggeration $(110 \%-140 \%)$ together, $F(1,223)=3.49$, $p=0.06$; however ratings of intelligence were significantly higher for exaggerated realistic characters than for exaggerated cartoon characters $F(1,218)=14.47, p=0.0002$. With slight exaggeration, $120 \%$, the realistic characters were significantly more likeable and intelligent than the cartoon characters, $F(1,217)=4.99, p=.03$ and $F(1,212)=16.05$, $p<0.0001$, respectively.

To investigate $\mathrm{H} 1 \mathrm{~b}$, we compared the likeability and intelligence ratings of damped $(60 \%$ - 90\%) cartoon characters to the ratings of damped realistic-looking characters, and we found that the ratings did not differ significantly, $F(1,223)=$ $.24, p=0.62$ and $F(1,218)=1.58, p=0.21$, respectively. When motion was noticeably damped $(60 \%$ and $70 \%)$, the cartoon characters were significantly less likeable than the realistic characters, $F(1,221)=4.89, p=0.0281$, confirming H1b for likeability when motion was extremely damped.

We expected that damped realistic characters would be more likeable and intelligent than exaggerated realistic characters (H1c); however, we found that slightly exaggerated $(110 \%$ and $120 \%)$ realistic characters were preferred 


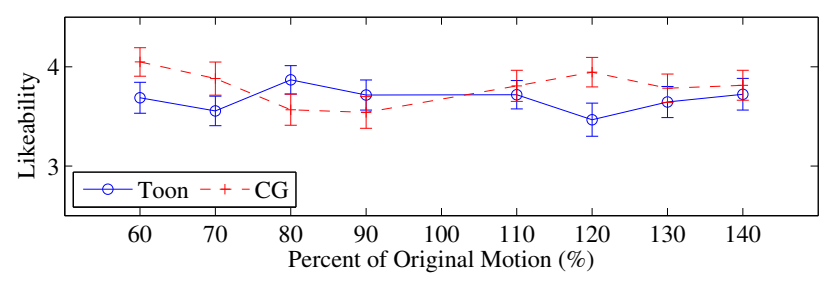

(a)

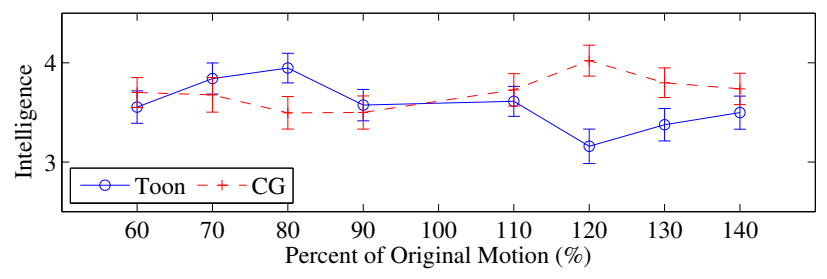

(b)

Fig. 4. Interaction effects on likeability and intelligence.

to slightly damped $(80 \%$ and $90 \%)$ realistic characters, $F(1,215)=4.95, p=0.03$ for likeability and $F(1,210)=$ $6.67, p=0.01$ for intelligence. We found similarly surprising results when investigating H1d: slightly damped cartoon characters were considered more intelligent than slightly exaggerated cartoon characters, $F(1,210)=7.20, p=0.008$.

Our unexpected results can partially be explained by the main effects of render style we found on both likeability and intelligence, $F(1,195)=4.44, p=0.037$ and $F(1,196)=$ $3.69, p=0.056$, respectively (see Fig. 5).

As hypothesized in $\mathrm{H} 2$, we found a significant main effect of motion level on perceptions of extraversion, as depicted in Fig. $6, F(7,197)=2.14, p=0.04$. A post hoc contrast verified that extraversion ratings did not differ significantly at the $60 \%$ and $70 \%$ levels, $F(1,198)=2.56, p=0.11$. There was a significant positive correlation between motion level and extraversion, $r=0.14, p=0.02$, suggesting that as motion level increases, perceptions of extraversion increase.

Although, we suspected (H3) that there would be a correlation between extraversion and intelligence as previous research had suggested [10], [13], [14], we found no such correlation. However, our data support hypothesis H4, that likeability and trustworthiness are positively correlated. The questions regarding likeability and trustworthiness all loaded onto the same factor during principal component analysis.

We were concerned that perceived character age could be confounded with rendering style, and specifically that realistic characters might be perceived as older than cartoon characters. We asked participants to estimate the age of the characters after they saw each one. We found no significant effect of rendering style on perceived character age, $F(1,25)=1.26, p=0.26$.

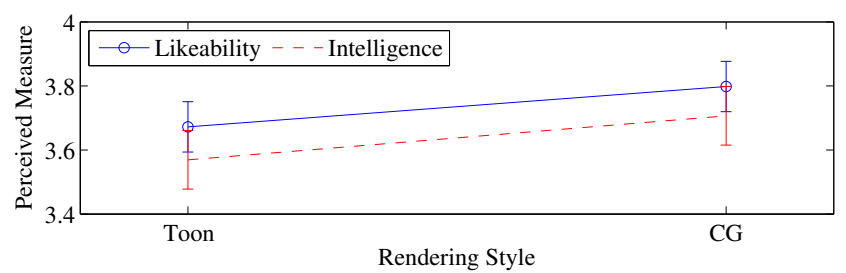

Fig. 5. Rendering Style Trends for Likeability and Intelligence

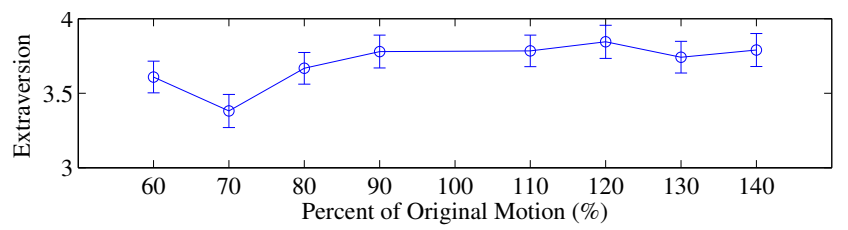

Fig. 6. Effect of motion level on extraversion.

\section{DISCUSSION}

If virtual humans, embodied agents, animated characters, and avatars could convey their personalities accurately and realistically, then they could be more effective at engaging, teaching, helping, and entertaining users. In this paper, we have examined the effects of rendering style and motion level on impressions of character personality. We wanted to know whether the character rendering style should match the amount of facial motion. We found evidence to support this idea when characters were extremely damped (60\% and $70 \%$ ) because the cartoon characters were then significantly less likeable than the realistic-looking characters.

Surprisingly, we also found support for a preference of characters with mismatched rendering style and motion level. Slightly exaggerated realistic-looking characters were more likeable and intelligent than slightly exaggerated cartoon characters. This result may be due to the fact that participants were more sensitive to slightly exaggerated motion on the realistic-looking character than on the cartoon character (see Fig. 3). The slight exaggeration of the realistic-looking character may have been perceived as the character being slightly more expressive, but the slight exaggeration in the cartoon character may have been unnoticed. Overly exaggerated characters did not affect participants' perceptions perhaps because the motion was perceived as unnatural, and the audio overpowered the visual information. Future research should investigate the interaction of facial movement and voice characteristics. Independent of motion level, our participants perceived the more realistic characters as more likeable and intelligent than the cartoon characters. The audio was unmodified and therefore may have may have been a better fit for the realistic-looking characters than the cartoon characters.

Previously, body motion speed and extraversion had been correlated; however, no previous studies investigated the relationship between the amount of facial motion and extraversion. We identified a positive relationship between these variables. Prior research was inconclusive in regards to the relationship between extraversion and intelligence, and we found no relationship; however, we did find a positive correlation between likeability and trustworthiness as expected.

We used rendering styles similar to the Toon Flat and Toon CG styles used by McDonnell and colleagues [9]. In contrast with their results, we found a significant difference between ratings of likeability and trustworthiness across the two styles. Our realistic characters were perceived as significantly 
more likeable and trustworthy than our cartoon characters. This difference may have been due to our animation technique as McDonnell and colleagues used full motion capture data to animate their characters.

\section{LIMITATIONS}

We conducted a controlled laboratory experiment to examine the relationship between rendering style and facial motion on viewers' perceptions of character personality. Unfortunately, we only had one actor and one actress, and therefore we could not investigate the effects of character gender on perceptions of character likeability, intelligence, and extraversion. Actor was perfectly confounded with character gender. We also used only two rendering styles. Future work should investigate the effects of character gender and more rendering styles by using more actors and characters.

We chose to use positive stories about ordinary situations. Context may have an important impact on perceptions of character personality. It is quite possible that exaggerating motion during expressions of negative emotion may have a different effect on participants' perceptions of personality.

We could not transfer the actors' motions to our character perfectly because 2D AAMs cannot measure motion accurately when the actors turn their heads or close their eyes and mouths completely. We requested that our actors limit their head motion while reading from a teleprompter. When the actors turned their heads slightly, this motion was translated into torso motion in the animated characters. The lack of head turns and the additional torso motion could have been perceived as unnatural. It is also unclear whether the facial motion maintains naturalness when we spatially modify it. There are many other animation techniques that could have been used, however we chose to use 2D AAMs because they are relatively quick and inexpensive to use. They are also customizable to individual people, so that subtle facial expressions are transferred to the animated characters.

\section{CONCLUSION}

Our experiment revealed that cartoon characters are less likeable than more realistic characters and that the effect is amplified when cartoon characters are presented with damped motion and more realistic characters are presented with exaggerated motion. These results suggest that if animators want to create intelligent cartoon characters, they should slightly damp the facial motion without worrying about making the character less likeable. If animators want to create likeable and intelligent realistic characters, they should use exaggerated facial motion. These results need to be validated with a larger study using more rendering styles, characters, and actors. Perceptions of extraversion were positively correlated with the amount of facial motion. This correlation implies that people could appear more extraverted than they really are by using avatars that exaggerate their motion. Animators who want to create extraverted characters should exaggerate the characters' facial expressions and analogously damp the characters' facial expressions to make their characters seem more introverted.

\section{ACKNOWLEDGEMENTS}

This study was funded by NSF grant CCF-0811450. We thank Brooke Kelly, Moshe Mahler, Tomas Simon, Iain Matthews, our actors, and Disney Research Pittsburgh staff, researchers, and interns for their help with this study.

\section{REFERENCES}

[1] A. Tartaro and J. Cassell, "Playing with virtual peers: Bootstrapping contingent discourse in children with autism," in ICLS, 2008, pp. 382389.

[2] J. Lasseter, "Principles of traditional animation applied to 3D computer animation," ACM SIGGRAPH Comput. Graph., vol. 21, no. 4, pp. 3544, Aug. 1987.

[3] F. Thomas and O. Johnston, Disney Animation: The Illusion of Life. Abbeville Press, 1981.

[4] G. Hodgkinson, "The seduction of realism," in ACM SIGGRAPH ASIA Educators Program, 2009, pp. 1-4.

[5] M. J. Gielniak and A. L. Thomaz, "Enhancing interaction through exaggerated motion synthesis," in HRI, 2012, pp. 375-382.

[6] T. Ribeiro and A. Paiva, "The illusion of robotic life: principles and practices of animation for robots," in HRI, 2012, pp. 383-390.

[7] L. Takayama, D. Dooley, and W. Ju, "Expressing thought: improving robot readability with animation principles," in HRI, 2011, pp. 69-76.

[8] K. M. Inkpen and M. Sedlins, "Me and my avatar: Exploring users comfort with avatars for workplace communication," in CSCW, 2011, pp. 383-386.

[9] R. McDonnell, M. Breidt, and H. H. Bülthoff, "Render me real?: investigating the effect of render style on the perception of animated virtual humans," ACM TOG, vol. 31, no. 4, pp. 91:1-91:11, Jul. 2012

[10] J. Wickett and P. Vernon, "Replicating the movement time-extraversion link ... with a little help from IQ," Pers. Indiv. Differ, vol. 28, no. 2, pp. 205-215, Feb. 2000.

[11] C. Doucet and R. M. Stelmack, "Movement time differentiates extraverts from introverts," Pers. Indiv. Differ, vol. 23, pp. 775-786, 1997.

[12] R. M. Stelmack, M. Houlihan, and P. A. McGarry-Roberts, "Personality, reaction time, and event-related potentials," JPSP, vol. 65, pp. 399-409, 1993.

[13] R. D. Roberts, "Fitts' law, movement time and intelligence," Pers. Indiv. Differ, vol. 23, pp. 227-246, 1997.

[14] M. B. Wolf and P. L. Ackerman, "Extraversion and intelligence: A meta-analytic investigation," Pers. Indiv. Differ., vol. 39, pp. 531-542, 2005

[15] S. L. Brodsky, T. M. Neal, R. J. Cramer, and M. H. Ziemke, "Credibility in the courtroom: How likeable should an expert witness be?" JAAPL, vol. 37, no. 4, pp. 525-532, 2009.

[16] S. Gosling, P. Rentfrow, and W. Swann, "A very brief measure of the big-five personality domains," J. Res. Pers., vol. 37, no. 6, pp. 504-528, 2003.

[17] D. H. Brainard, "The psychophysics toolbox," Spatial Vision, vol. 10, pp. 433-436, 1997.

[18] D. Pelli, "The videotoolbox software for visual psychophysics: Transforming numbers into movies." Spatial Vision, vol. 10, pp. 437-442, 1997.

[19] M. Kleiner, D. Brainard, and D. Pelli, "What's new in psychtoolbox3?" in Perception, vol. 36. ECVP Abstract Supplement, 2007.

[20] T. Cootes, G. Edwards, and C. Taylor, "Active appearance models," IEEE PAMI, vol. 23, no. 6, pp. 681-685, 2001.

[21] T. Cootes, G. Wheeler, K. Walker, and C. Taylor, "View-based active appearance models," Image Vision Comput., vol. 20, no. 910, pp. 657 $-664,2002$.

[22] I. Matthews and S. Baker, "Active appearance models revisited," IJCV, vol. 60, pp. 135-164, 2004.

[23] S. M. Boker, J. Cohn, B. Theobald, I. Matthews, T. Brick, and J. Spies, "Effects of damping head movement and facial expression in dyadic conversation using real-time facial expression tracking and synthesized avatars," Phil. Trans. R. Soc. B, vol. 364, pp. 3485-3495, Dec. 2009

[24] B.-J. Theobald, I. Matthews, M. Mangini, J. R. Spies, T. R. Brick, J. F. Cohn, and S. M. Boker, "Mapping and manipulating facial expression," Lang. Speech, vol. 52, pp. 369-386, 2009.

[25] J. R. Lewis, "Pairs of latin squares to counterbalance sequential effects and pairing of conditions and stimuli," in Proc. Hum. Fact. Soc. An., 1989, pp. 1223-1227. 